Relations industrielles

Industrial Relations

\title{
Le mouvement des salaires dans la Province de Québec
}

\section{Yves Urbain}

Volume 9, numéro 3, juin 1954

URI : https://id.erudit.org/iderudit/1022875ar

DOI : https://doi.org/10.7202/1022875ar

Aller au sommaire du numéro

\section{Éditeur(s)}

Département des relations industrielles de l'Université Laval

ISSN

0034-379X (imprimé)

1703-8138 (numérique)

Découvrir la revue

\section{Citer ce document}

Urbain, Y. (1954). Le mouvement des salaires dans la Province de Québec. Relations industrielles / Industrial Relations, 9(3), 297-298.

https://doi.org/10.7202/1022875ar
Résumé de l'article

Dans une conférence qu'il prononçait devant un groupe de patrons à Montréal le 10 mai 1954, le professeur Yves Urbain a envisagé le problème très important des différentiels de salaire dans la Province de Québec. Il en conclut à une hausse inévitable. Après avoir expliqué les raisons historiques de l'infériorité économique des entreprises canadiennes-françaises, il poursuit son exposé.
Tous droits réservés ( Département des relations industrielles de l’Université Laval, 1954
Ce document est protégé par la loi sur le droit d'auteur. L'utilisation des services d'Érudit (y compris la reproduction) est assujettie à sa politique d'utilisation que vous pouvez consulter en ligne.

https://apropos.erudit.org/fr/usagers/politique-dutilisation/ 


\section{LE MOUVEMENT DES SALAIRES DANS LA PROVINCE DE QUEBEC}

\section{Yves Urbain}

Dans une conférence qu'il prononçait devant un groupe de patrons à Montréal le 10 mai 1954, le professeur Yves Urbain a envisagé le problème très important des différentiels de salaire dans la Province de Québec. Il en conclut à une hausse inévitable. Après avoir expliqué les raisons historiques de l'infériorité économique des entreprises canadiennes-françaises, il poursuit son exposé.

Prendre les devants

Il naît de cette situation, quelques problèmes gros de conséquences. Pour se perpétuer, la culture canadienne-française doit disposer d'une hiérarchie d'élites financières, industrielles, techniques et ouvrières capable, dans le régime de concurrence qui existe, d'emporter en nombre suffisant les postes-clé de l'économie québécoise. Or, le passé ne prépare guère les Canadiens français à ces fonctions. Comme il n'y a aucun doute que la Province soit orientée pour toujours vers l'industrie, il ne peut être question, pour les habitants de ce pays de s'accrocher à leur vocation principalement rurale du passé, il faut pouvoir prendre les devants dans l'industrialisation.

L'impréparation industrielle dont il vient d'être fait état a contenu la plupart des dirigeants canadiens-français dans des entreprises plutôt moyennes et petites, dans des secteurs d'activité à équipement plus réduit, en un mot dans des directions économiques ou bien moins profitables ou bien sujettes à la concurrence victorieuse des dirigeants d'origine canadienne-française. Dans cette structure, la question des salaires et des charges sociales a pu apparaître au premier plan des préoccupations des dirigeants canadiens-français et ceux-ci ont pu voir dans la hausse des salaires et des charges sociales, la cause, la cause évitable de leurs difficultés et, en fin de compte, l'objet principal de leur application. Ce serait, de ma part, faillir au devoir d'impartialité, qui est le premier des devoirs de l'intellectuel, que de ne pas signaler l'erreur que contient le raisonnement que je viens de retracer. Ainsi que la logique et l'expérience le démontrent, dans tous les pays où l'industrialisation s'est répandue les salaires se sont élevés inéluctablement, cette hausse a gagné successivement tous les cantons après avoir frappé les villes et elle s'est développée jusqu'au niveau correspondant à la productivité du travail dans l'économie nationale, jusqu'au niveau correspondant à la productivité du travail atteinte dans le Continent ou dans le monde si on considère séparément les industries. Or, qu'est-ce que l'économie nationale ou continentale pour la province de Québec? c'est le Canada tout entier, c'est l'Amérique du Nord. Pour autant que l'industrialisation de la province de Québec se poursuive, et elle doit se poursuivre si l'on veut maintenir le bloc démographique canadien-français il faut s'attendre à une hausse des salaires vers les niveaux atteints dans l'ensemble du Canada et, pour certaines branches d'industrie, vers les niveaux atteints dans les Etats-Unis voisins. Sous peine de concentrer toute la population dans lune ou l'autre grande ville et, sous peine, de voir se tarir la population des campagnes, il devra se produire, dans tous les cantons, une hausse de salaire qui, à l'avenir, sera peut-être plus que proportionnelle à celle qu'on enregistrera dans les centres. 


\section{Hausse inévitable des Salaires}

Sans doute, le mouvement de hausse des salaires dont les caractéristiques viennent d'être décrites, pourra être retardé de ci de là, sous l'influence de circonstances économiques ou sociales passagères; mais, pour les mêmes raisons, il pourra s'accélérer à d'autres moments. En fin de compte, il est inévitable.

Une circonstance inévitable comme ce mouvement de hausse des salaires n'étant que passagèrement différable, doit être considérée, comme un fait, comme une donnée du problème qui se pose aux dirigeants, comme un point dont il faut partir si l'on veut aboutir. C'est là, à mon sens, qu'un mouvement patronal peut intervenir et se rendre grandement utile dans une oeuvre absolument essentielle: celle de la formation sociale et personnelle des dirigeants financiers et industriels, formation sans laquelle il n'est pas possible d'entrevoir la solution des problèmes qui se posent à vous aujourd'hui, pris que vous êtes dans une transformation industrielle en grande partie venue du dehors et réalisée par les méthodes du dehors.

Dans cette oeuvre de formation personnelle et sociale du dirigeant, le mouvement se devrait d'être un unitiateur, un promoteur, quitte à abandonner telle ou telle partie de cette formation aux institutions les plus propres à réaliser l'éducation souhaitée. Entendue de la sorte, laction du mouvement serait une action de recherches, d'opinion autant ou plus qu'une action d'exécution.

\section{LE REGLEMENT DES CONFLITS DE DROIT}

Les 3 et 4 mai dernier, se réunissaient au 9è Congrès des Relations Industrielles organisé par le Département des Relations Industrielles de l'Université Laval un nombre imposant de représentants de tous les milieux du travail. Tous, directement ou indirectement intéressés par les problèmes industriels, ont contribué, soit au cours des conférences, soit au cours des délibérations, à rendre intéressant ce congrès dont le thème était: «Le règlement des conflits de droit». Les chiffres suivants illustrent exactement la répartition des congressistes:

\section{Distribution des délégués}

Entreprises et associations patronales...

$\begin{array}{llllllllllll}\text { Syndicats ouvriers } \ldots . . & \ldots & \ldots & \ldots & \ldots & \ldots & \ldots & \ldots & \ldots & \ldots & \ldots & \ldots .\end{array} \quad 170$

Gouvernements fédéral et provincial, municipalités ... .... 84

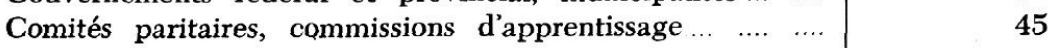

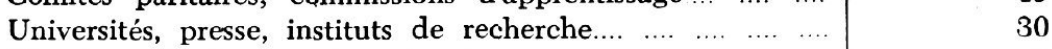

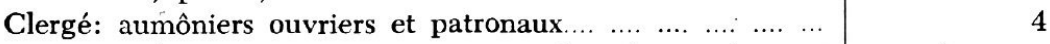

